\title{
Development of a recycling process for an industrial scale production of tipifarnib
}

Xiaohu Deng ${ }^{\S}$, Pingda Ren ${ }^{\S}$, Wanping Mai ${ }^{\S}$, Thomas Fu ${ }^{\S}$, Hang Zhang ${ }^{\dagger}$, Xianfeng

$$
\mathrm{Cai}^{\dagger}, \text { Lu Wang }{ }^{\dagger}
$$

§Kura Oncology Inc., 12730 High Bluff Drive, Suite 400, San Diego, CA 92130, USA

†STA Pharmaceutical Co., Ltd., ("WuXi STA"), a WuXi AppTec company, 90 Delin Road, Waigaoqiao Pilot Free Trade Zone, Shanghai, 200131, China

* Currently at Vividion Therapeutics Inc.; ID: orcid.org/0000-0003-1393-4446;

Email: xdeng1000@yahoo.com

Notes: The authors declare no competing financial interest. 
${ }^{1} \mathrm{H}$ NMR for compound 7

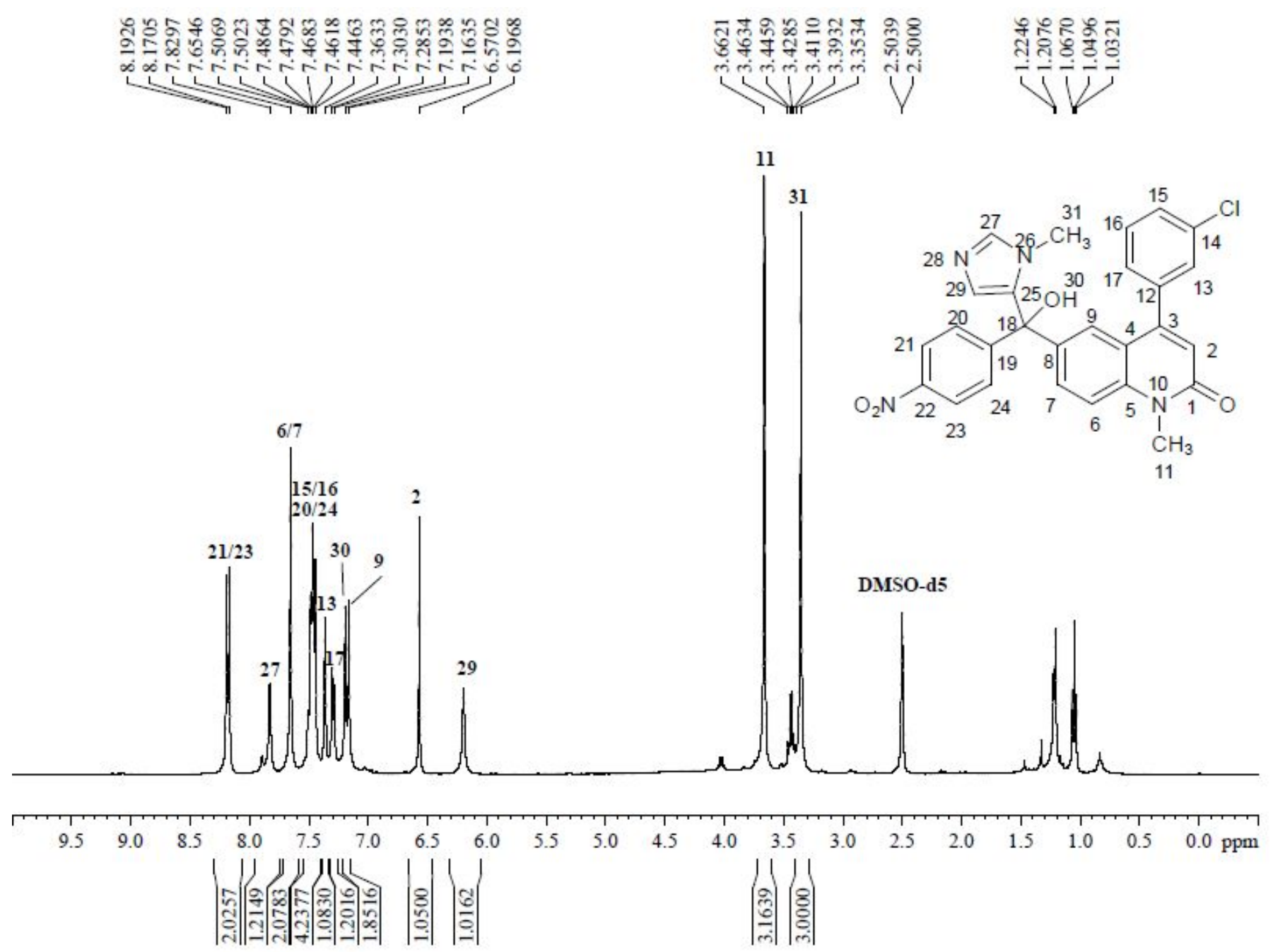


${ }^{13} \mathrm{C}$ NMR for compound 7
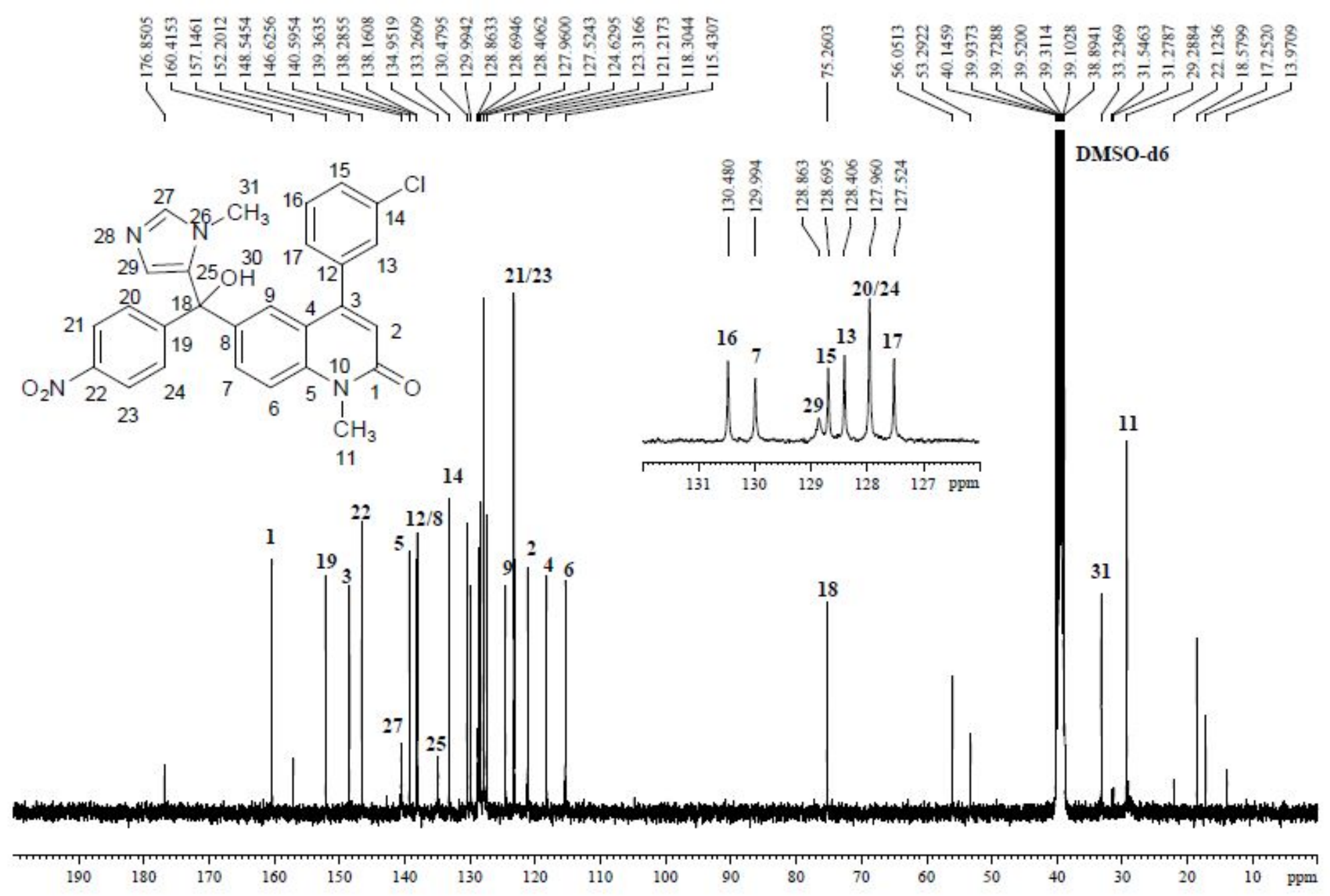

$20 \quad 10 \mathrm{ppm}$ 
IR for compound 7

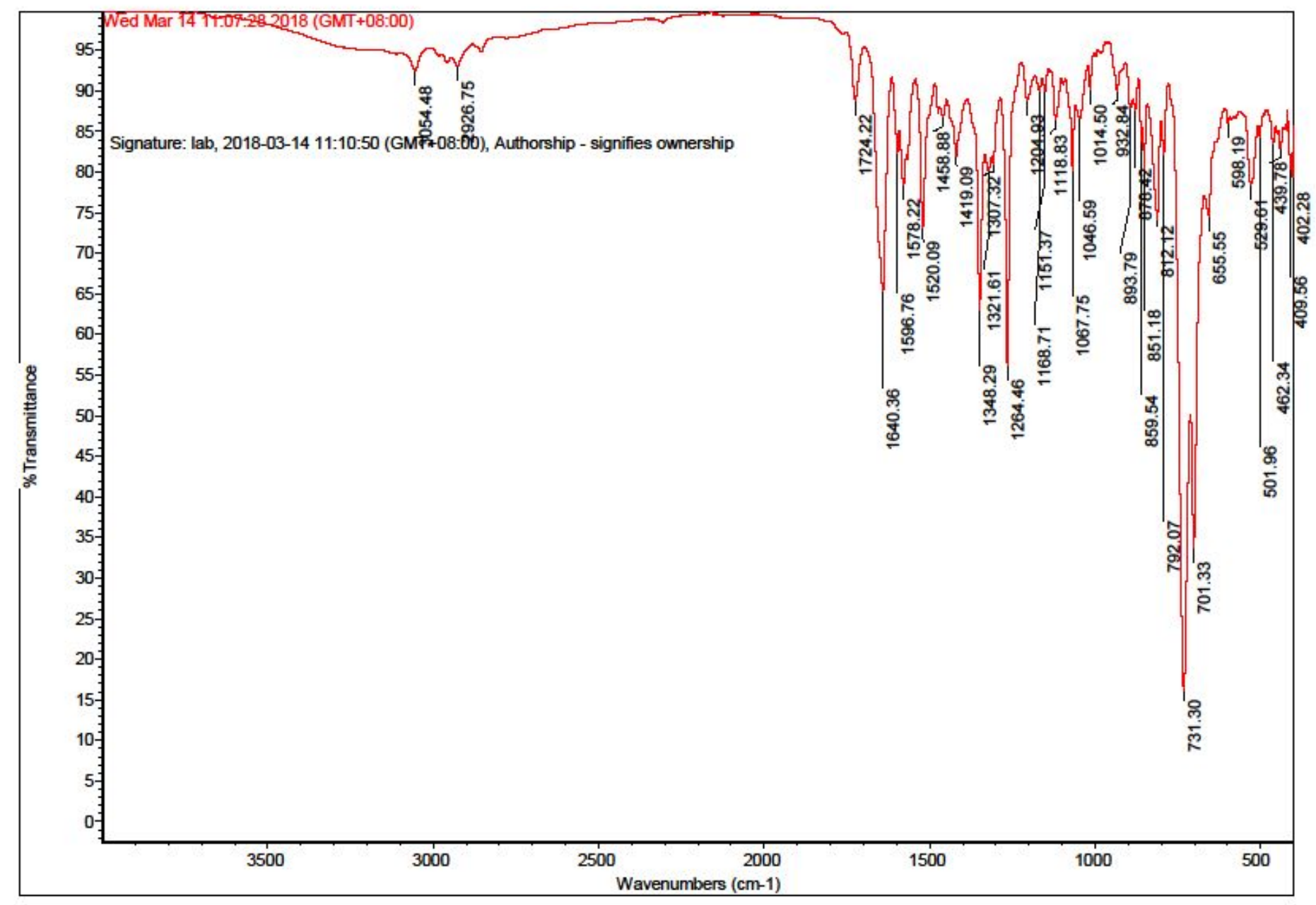




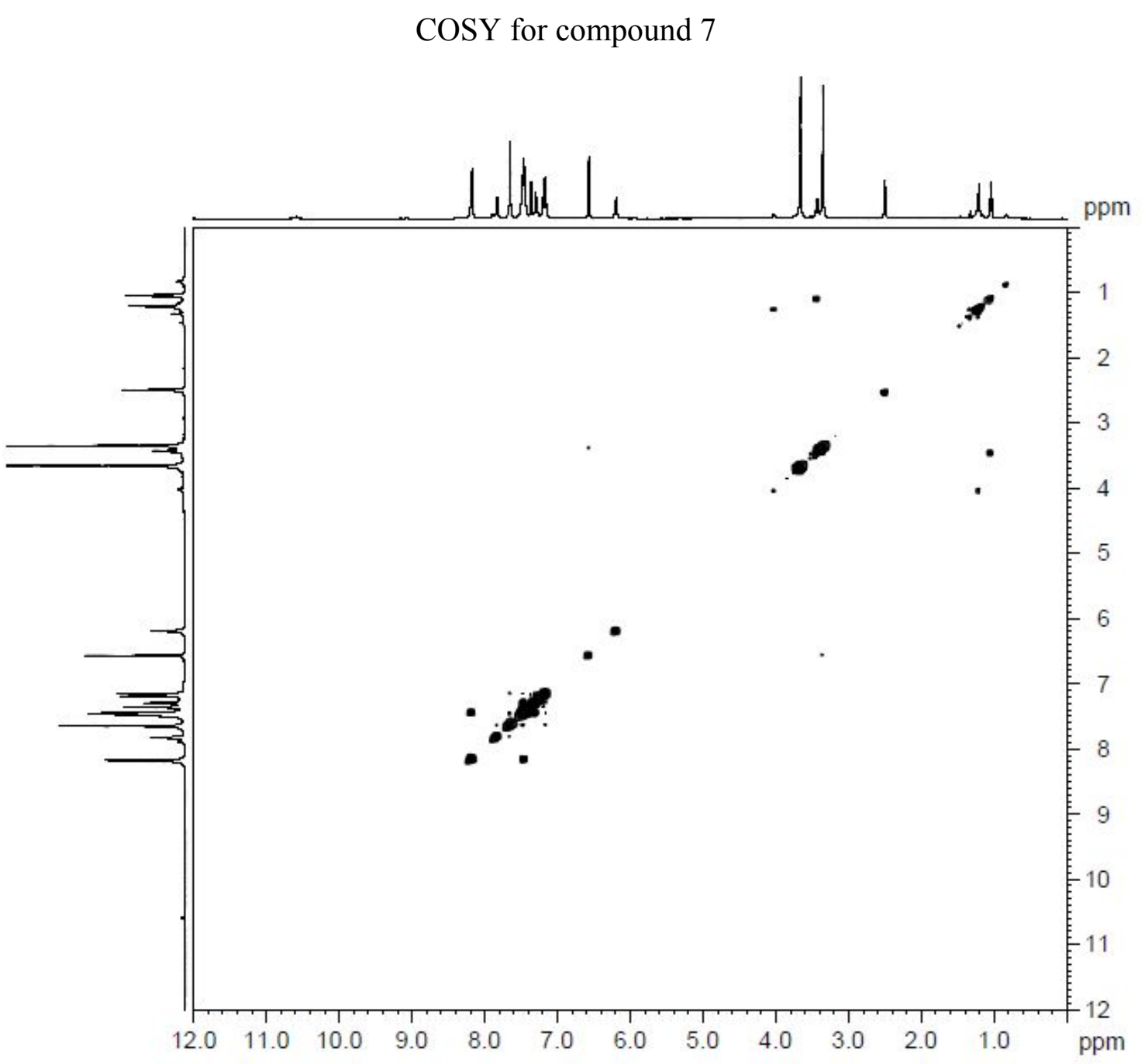


HMBC for compound 7

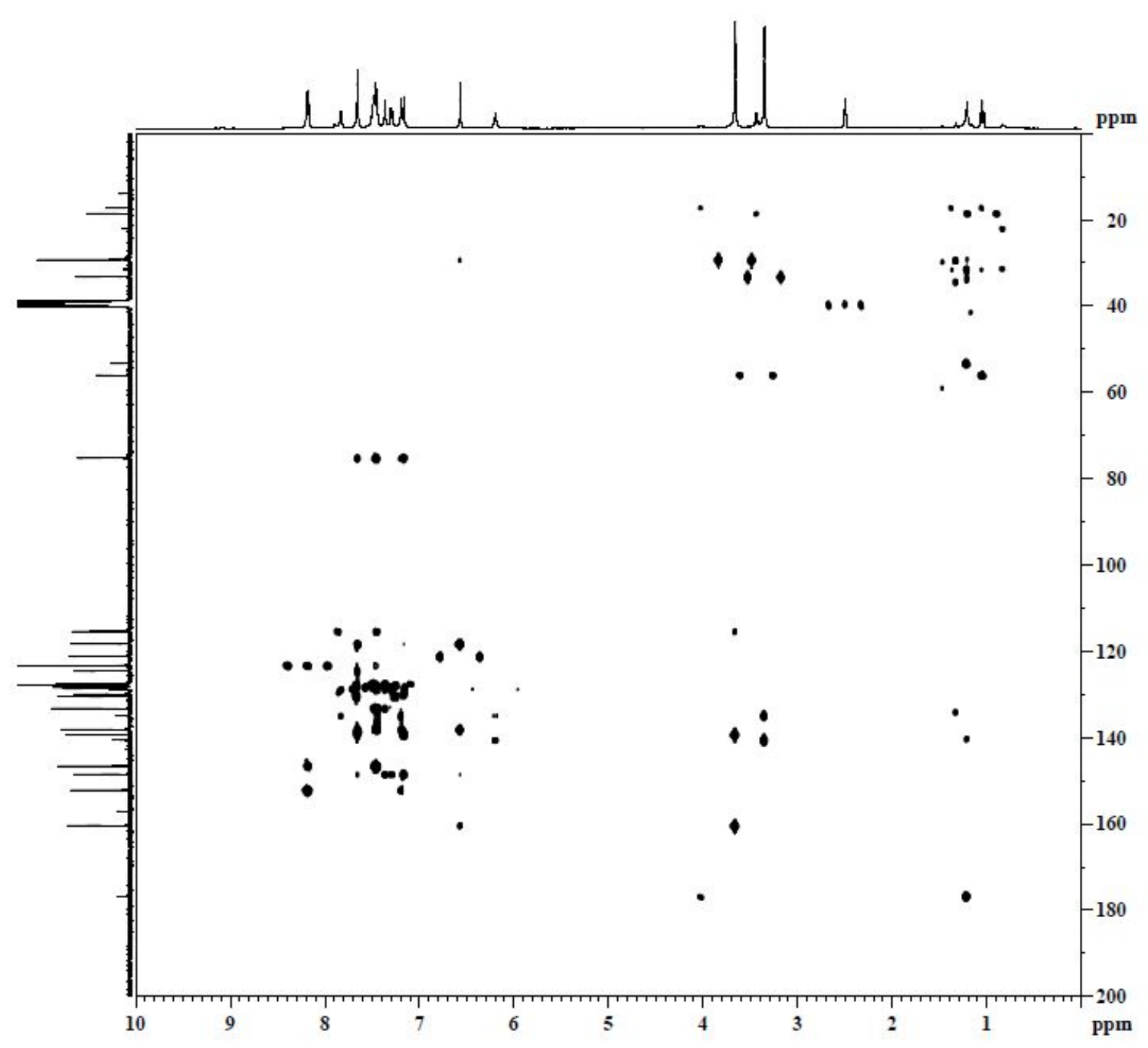




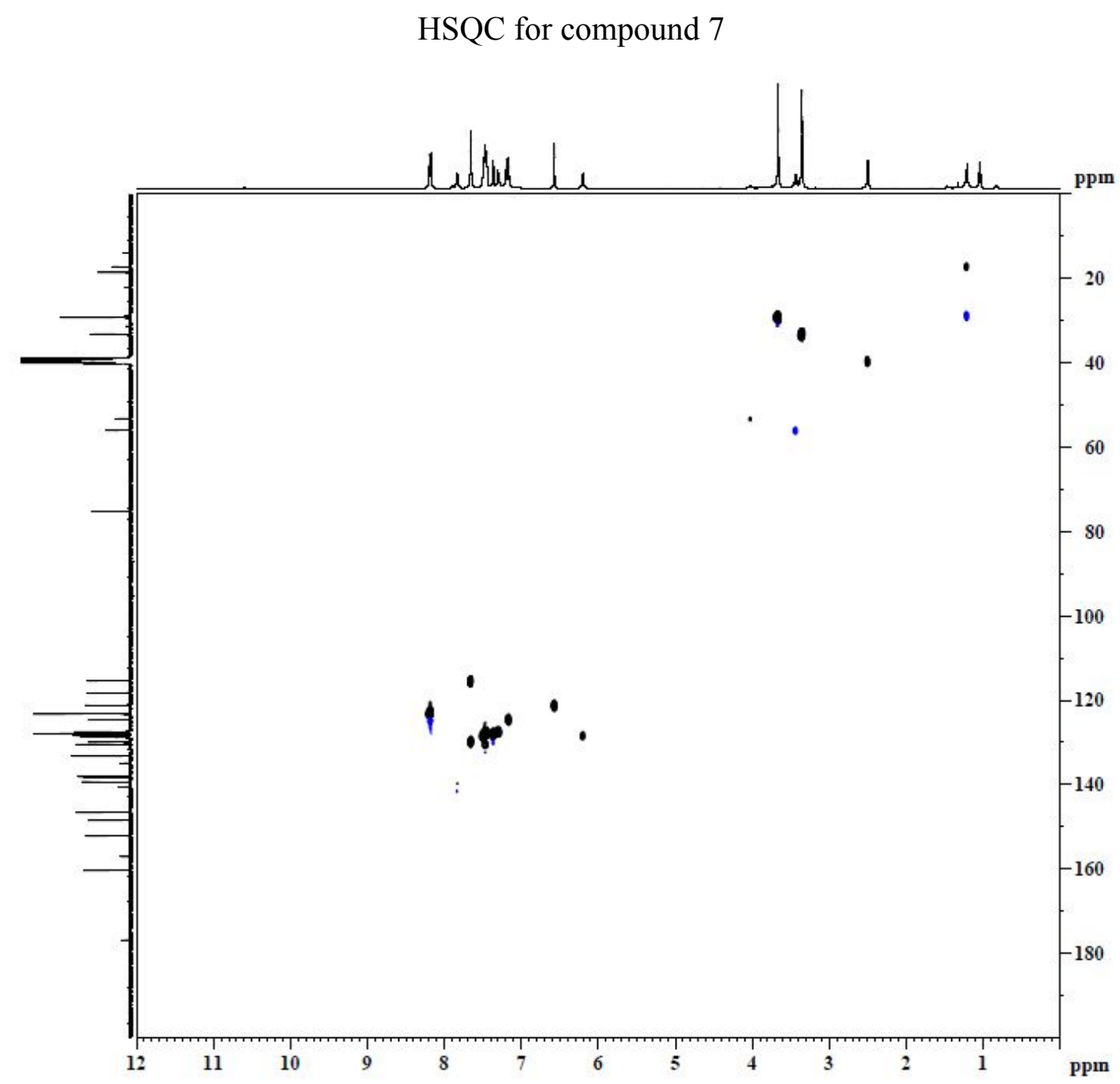


Reaction profile of $\mathrm{NaNO}_{2}$-mediated racemzaiton step (scheme 4, crude after workup)

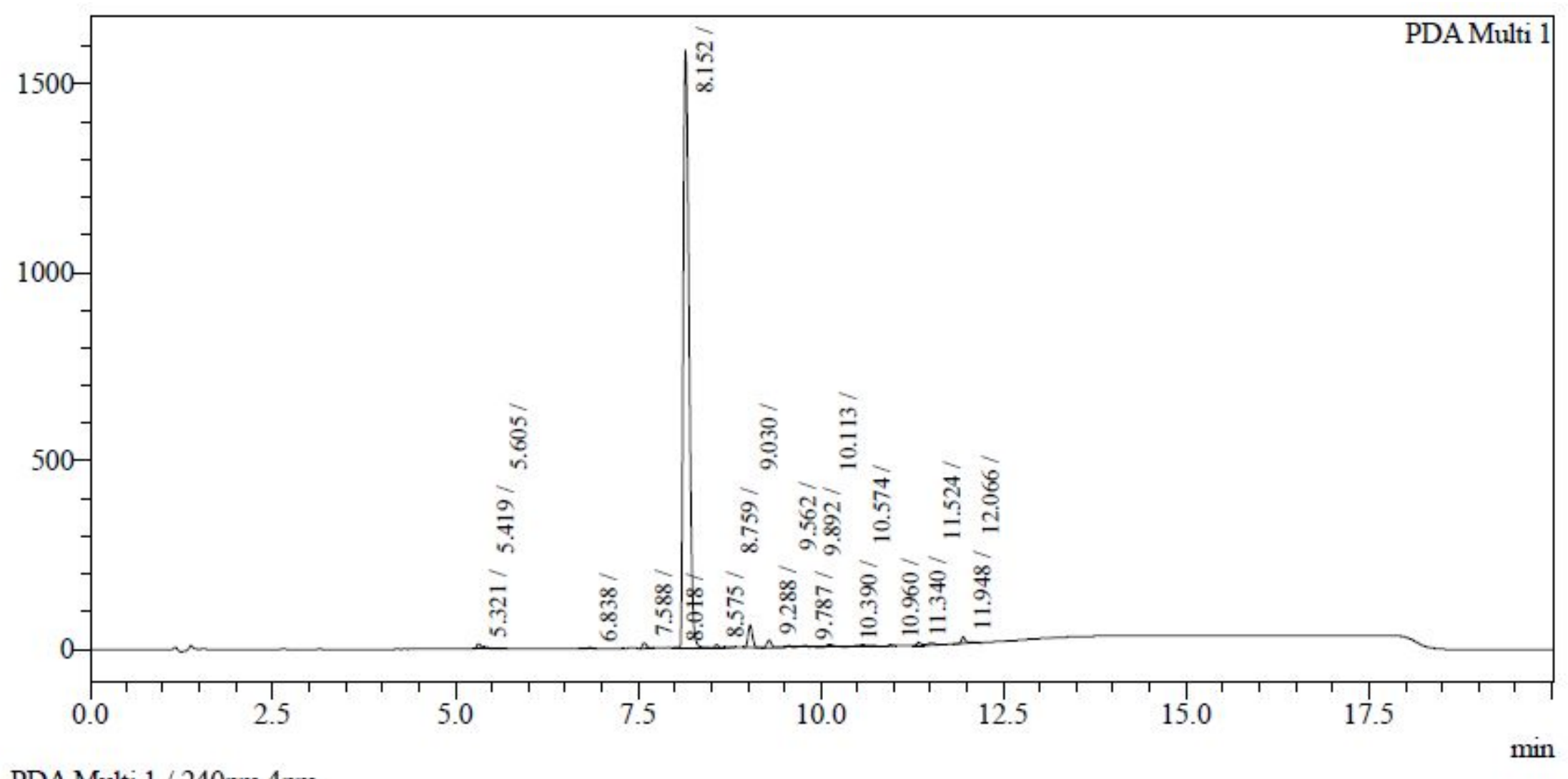

L PDA Multi $1 / 240 \mathrm{~nm}, 4 \mathrm{~nm}$ 
<Results>

PDA Ch1 240nm

\begin{tabular}{|c|c|c|c|c|c|c|c|}
\hline Peak\# & Name & Ret. Time & Area & Area $\%$ & Height & Resolution & Tailing Factor \\
\hline 1 & & 5.321 & 61733 & 0.675 & 14673 & 0.0 & 0.0 \\
\hline 2 & & 5.419 & 22506 & 0.246 & 5095 & 0.6 & 0.0 \\
\hline 3 & & 5.605 & 865 & 0.009 & 211 & 1.1 & 0.0 \\
\hline 4 & Compound 1 & 6.838 & 13598 & 0.149 & 3888 & 10.7 & 1.2 \\
\hline 5 & & 7.588 & 55827 & 0.611 & 14487 & 6.7 & 1.3 \\
\hline 6 & & 8.018 & 8224 & 0.090 & 2745 & 4.2 & 1.0 \\
\hline 7 & Compound 2 & 8.152 & 8291828 & 90.720 & 1586753 & 1.1 & 1.4 \\
\hline 8 & & 8.575 & 32983 & 0.361 & 8783 & 3.2 & 1.3 \\
\hline 9 & & 8.759 & 5775 & 0.063 & 1522 & 1.6 & 0.0 \\
\hline 10 & & 9.030 & 235963 & 2.582 & 58901 & 2.3 & 1.2 \\
\hline 11 & & 9.288 & 79435 & 0.869 & 19669 & 2.1 & 1.2 \\
\hline 12 & & 9.562 & 15523 & 0.170 & 4045 & 2.3 & 0.0 \\
\hline 13 & & 9.787 & 31092 & 0.340 & 5114 & 1.8 & 0.0 \\
\hline 14 & & 9.892 & 7507 & 0.082 & 1812 & 0.7 & 0.0 \\
\hline 15 & & 10.113 & 43461 & 0.475 & 6263 & 1.4 & 1.6 \\
\hline 16 & & 10.390 & 3288 & 0.036 & 740 & 1.8 & 0.0 \\
\hline 17 & & 10.574 & 53779 & 0.588 & 5666 & 1.3 & 1.5 \\
\hline 18 & & 10.960 & 10408 & 0.114 & 2990 & 2.9 & 1.0 \\
\hline 19 & & 11.340 & 31513 & 0.345 & 7743 & 3.4 & 0.0 \\
\hline 20 & & 11.524 & 35933 & 0.393 & 5569 & 1.2 & 0.0 \\
\hline 21 & & 11.948 & 79763 & 0.873 & 17929 & 2.7 & 0.0 \\
\hline 22 & & 12.066 & 19066 & 0.209 & 3421 & 0.5 & 0.0 \\
\hline Total & & & 9140069 & 100.000 & 1778021 & & \\
\hline
\end{tabular}


Reaction profile of the $\mathrm{NaNO}_{2}$-free racemzaiton step (scheme 5)

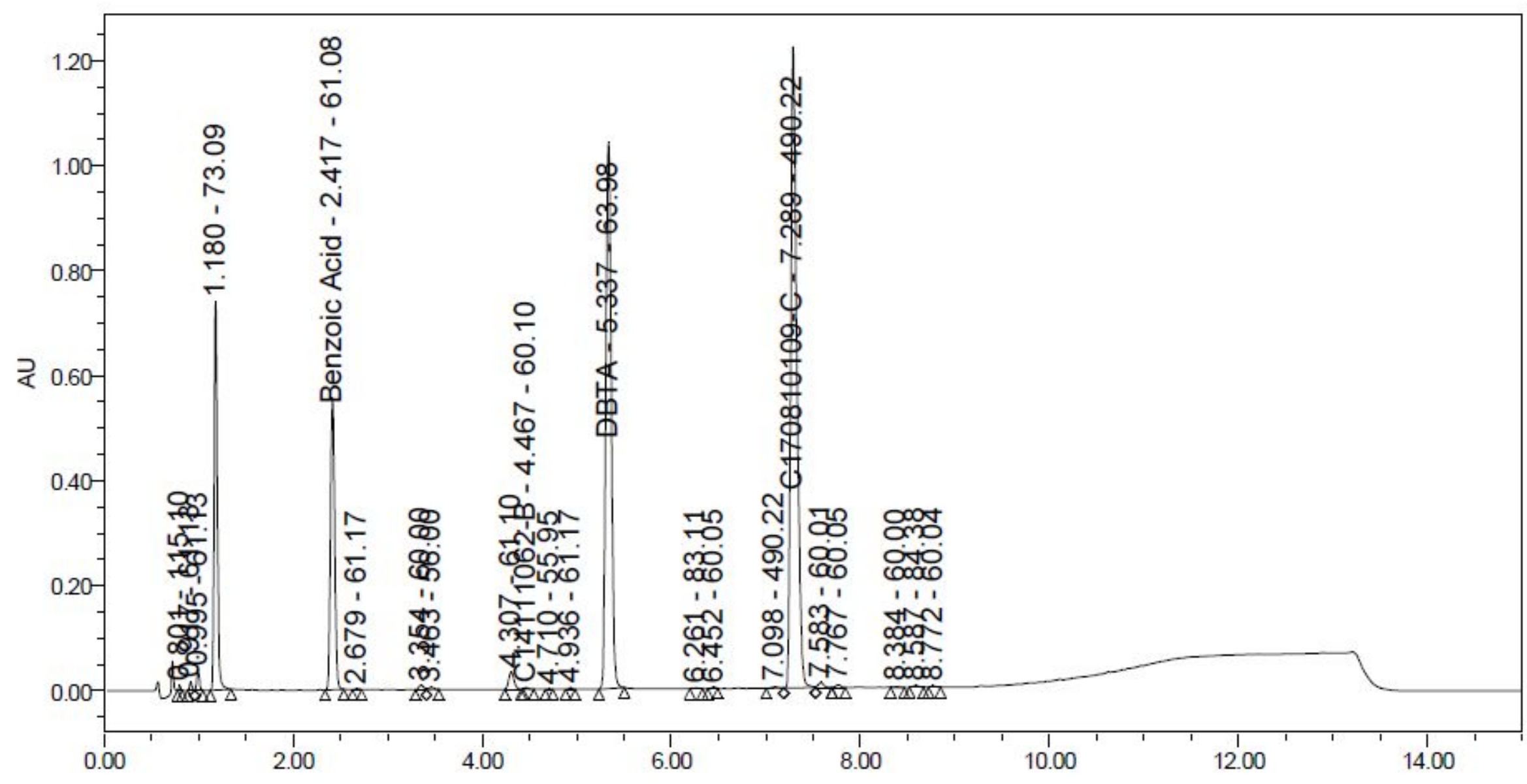




\begin{tabular}{|l|l|c|r|r|r|l|l|}
\hline & Peak Name & RT & Area & $\%$ Area & Height & USP Resolution & USP Tailing \\
\hline 1 & & 0.801 & 12586 & 0.09 & 8920 & & \\
\hline 2 & & 0.917 & 28314 & 0.21 & 15416 & & \\
\hline 3 & & 0.995 & 71748 & 0.53 & 37355 & & \\
\hline 4 & & 1.180 & 1731064 & 12.88 & 739311 & & \\
\hline 5 & Benzoic Acid & 2.417 & 1724545 & 12.83 & 555579 & & \\
\hline 6 & & 2.679 & 6913 & 0.05 & 2345 & & \\
\hline
\end{tabular}




\begin{tabular}{|c|c|r|r|r|r|l|l|}
\hline & Peak Name & RT & \multicolumn{1}{c|}{ Area } & $\%$ Area & Height & USP Resolution & USP Tailing \\
\hline 7 & & 3.354 & 30469 & 0.23 & 8476 & & \\
\hline 8 & & 3.463 & 17214 & 0.13 & 4259 & & \\
\hline 9 & & 4.307 & 126585 & 0.94 & 32665 & & \\
\hline 10 & C14111062-B & 4.467 & 7104 & 0.05 & 2679 & & \\
\hline 11 & & 4.710 & 3035 & 0.02 & 1206 & & \\
\hline 12 & & 4.936 & 8866 & 0.07 & 2999 & & \\
\hline 13 & DBTA & 5.337 & 4123921 & 30.68 & 1040821 & & \\
\hline 14 & & 6.261 & 5940 & 0.04 & 1626 & & \\
\hline 15 & & 6.452 & 7123 & 0.05 & 2369 & & \\
\hline 16 & & 7.098 & 10501 & 0.08 & 2587 & & \\
\hline 17 & C170810109-C & 7.289 & 5441712 & 40.49 & 1221346 & & \\
\hline 18 & & 7.583 & 39324 & 0.29 & 9742 & & \\
\hline 19 & & 7.767 & 17101 & 0.13 & 4928 & & \\
\hline 20 & & 8.384 & 5808 & 0.04 & 1558 & & \\
\hline 21 & & 8.587 & 13036 & 0.10 & 3247 & & \\
\hline 22 & & 8.772 & 8032 & 0.06 & 2257 & & \\
\hline
\end{tabular}


HPLC of the isolated recycled compound 2 with $\mathrm{NaNO}_{2}$-free process

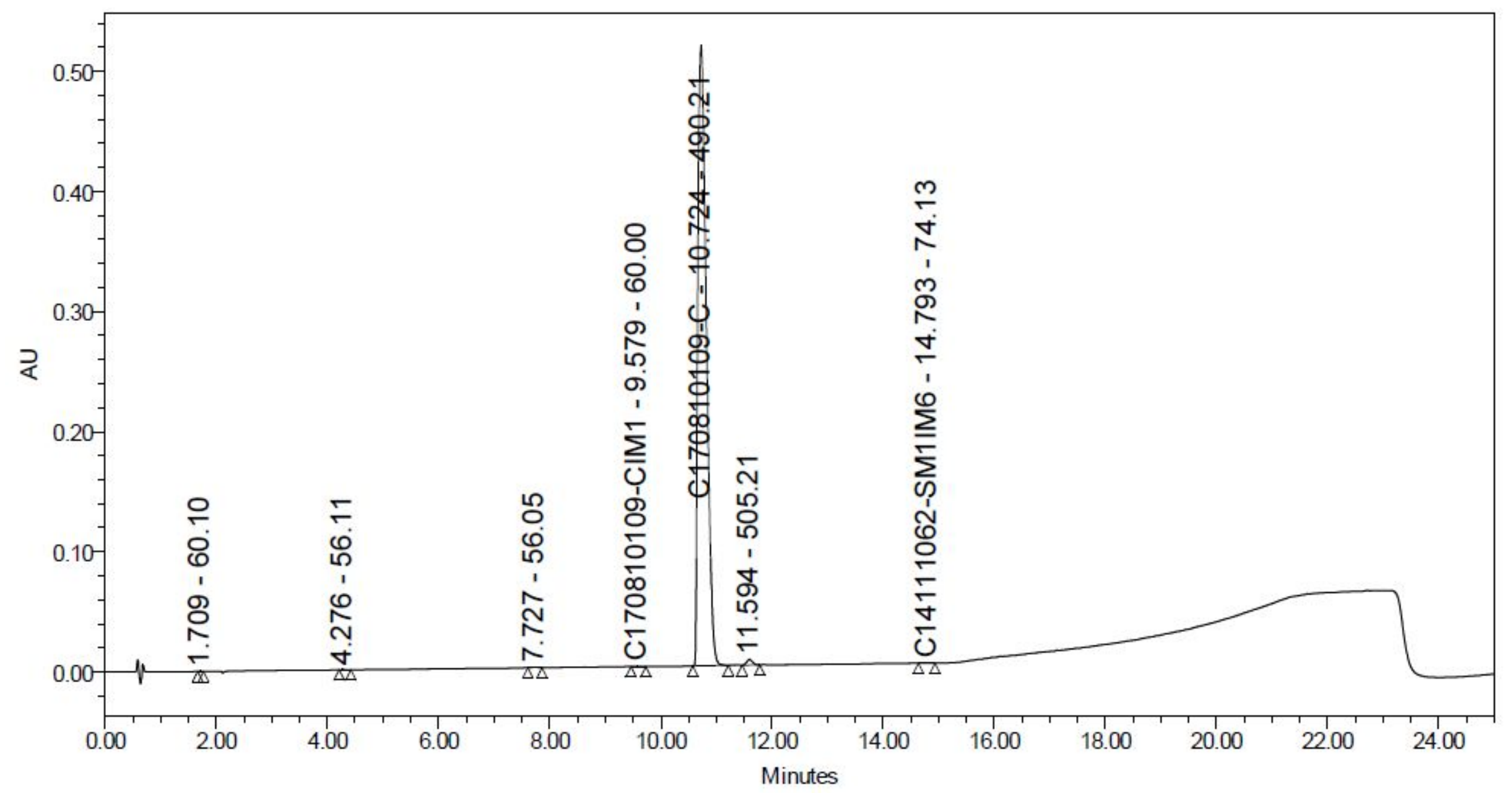




\begin{tabular}{|c|c|c|c|c|c|c|c|}
\hline & Peak Name & RT & Area & $\%$ Area & Height & USP Resolution & USP Tailing \\
\hline 1 & & 1.709 & 1385 & 0.02 & 609 & & \\
\hline 2 & & 4.276 & 1449 & 0.03 & 282 & & \\
\hline 3 & & 7.727 & 3254 & 0.06 & 533 & & \\
\hline 4 & C170810109-CIM1 & 9.579 & 3056 & 0.05 & 468 & & \\
\hline 5 & C170810109-C & 10.724 & 5512427 & 99.20 & 516416 & & \\
\hline 6 & & 11.594 & 32917 & 0.59 & 4564 & & \\
\hline 7 & C14111062-SM1IM6 & 14.793 & 2175 & 0.04 & 232 & & \\
\hline
\end{tabular}


${ }^{1} \mathrm{H}$ NMR for Recycled compound 2

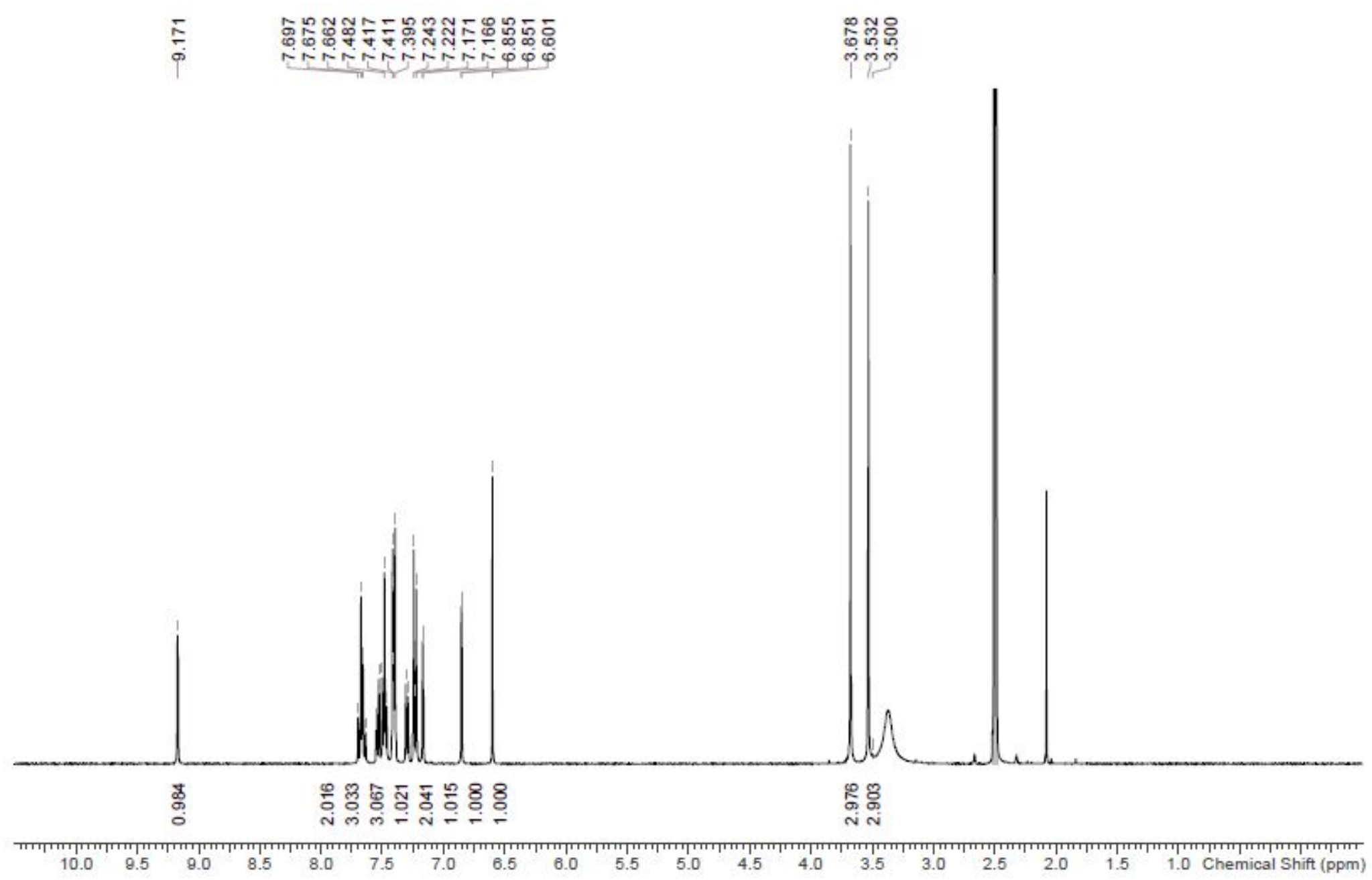


${ }^{13} \mathrm{C}$ NMR for Recycled compound 2

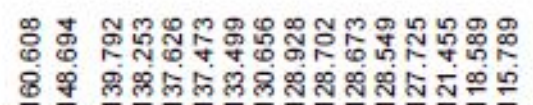

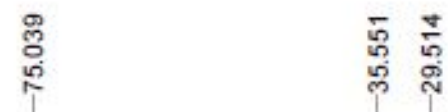

1 -........

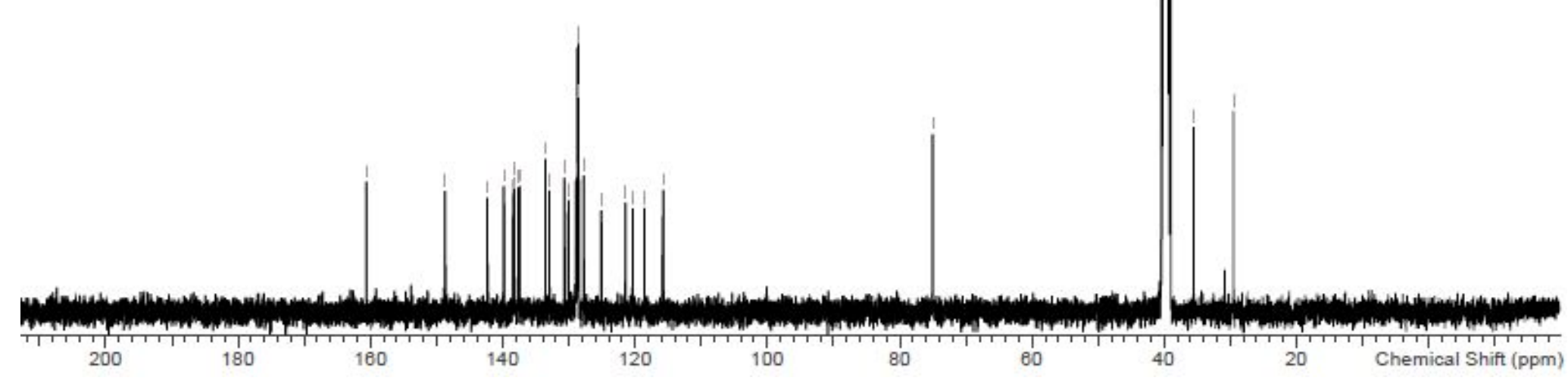

